\title{
Percutaneous Drainage Combined with Hyperbaric Oxygen Therapy for Pyogenic Spondylitis with Iliopsoas Abscess
}

\author{
Katsuhiro Tofuku ${ }^{1}$, Hiroaki Koga ${ }^{1}$, Setsuro Komiya ${ }^{2}$ \\ ${ }^{1}$ Department of Orthopaedic Surgery, Imakiire General Hospital, Kagoshima, Japan \\ ${ }^{2}$ Department of Orthopaedic Surgery, Kagoshima Graduate School of Medical and Dental Sciences, Kagoshima, Japan
}

\begin{abstract}
Study Design: A retrospective study.
Purpose: The purpose of this study was to evaluate outcomes in patients with pyogenic spondylitis accompanied by iliopsoas abscess who were treated by percutaneous drainage combined with hyperbaric oxygen (HBO) therapy.

Overview of Literature: To the best of our knowledge, there have been no previous reports of the use of percutaneous drainage combined with HBO therapy for the treatment of this condition.

Methods: Twenty-three patients (13 men, 10 women; mean age, 69.0 years; range, 45-85 years) were treated with percutaneous drainage combined with HBO therapy in addition to commonly used conservative therapy. Mean follow-up duration was 27.7 months (range, 12-48 months). Clinical outcomes and imaging examinations were retrospectively investigated.

Results: Symptoms such as low back pain, radicular pain, and hip pain resolved in all patients immediately after treatment. Mean time from the start of treatment to the return of $\mathrm{C}$-reactive protein levels to normal or baseline values recorded before the onset of spondylitis was 28.3 days (range, 8-56 days). In the final set of follow-up radiographic studies, all patients were free from progressive destructive changes. Follow-up magnetic resonance images or computed tomography with contrast enhancement confirmed the disappearance or near-total resolution of the iliopsoas abscess cavity with healing of the pyogenic spondylitis in all 23 patients. No recurrences were observed during follow-up.

Conclusions: The present study suggests that patients with pyogenic spondylitis accompanied by iliopsoas abscess can be cured without a prolonged period of therapy or recurrence using this treatment.
\end{abstract}

Keywords: Psoas abscess; Spondylitis; Hyperbaric oxgen therapy; Drainage

\section{Introduction}

The rate of occurrence of pyogenic spondylitis has been increasing among patients who are immunocompromised due to severe underlying diseases such as diabetes mellitus and malignant tumor as well as among patients undergoing chronic hemodialysis. Iliopsoas abscesses sometimes develop in patients with pyogenic spondylitis. Although small iliopsoas abscesses usually respond to ordinary conservative treatment, cases of pyogenic spondylitis accompanied by larger abscesses are, in general, difficult to cure using conservative methods. Because most of

Received Jul 5, 2013; Revised Aug 12, 2013; Accepted Aug 13, 2013

Corresponding author: Katsuhiro Tofuku

Department of Orthopaedic Surgery, Imakiire General Hospital,

4-16 Shimotatsuo, Kagoshima 892-8502, Japan

Tel: +81-99-226-2211, Fax: +81-99-222-7906, E-mail: tofuku@m2.kufm.kagoshima-u.ac.jp 
these cases occur in immunocompromised patients who are in poor general condition, a less invasive operative procedure is desirable. Although percutaneous drainage has been reported to be effective in iliopsoas abscesses secondary to pyogenic spondylitis [1], valid long-term results of this technique are not yet available. Drainage alone for a secondary psoas abscess may result in significant recurrence rates, and percutaneous drainage alone is not always sufficient for the treatment of secondary iliopsoas abscesses due to pyogenic spondylitis [2-5]. In addition, some authors have emphasized the role of the spine as the primary source of infection for secondary iliopsoas abscess and have suggested that it is essential to combine abscess drainage with curative treatment of the primary focus of infection [6].

Several authors have described minimally invasive techniques that address spondylitis as the primary focus for the treatment of this condition [7-10]. Although the clinical results have been satisfactory in these studies, the numbers of patients were small, and the techniques are technically demanding and sometimes time-consuming, which may present obstacles to these techniques becoming accepted as standard procedures. Simpler and easier treatment for this condition is still needed. Hyperbaric oxygen (HBO) is now a recognized treatment for several disorders, including gas gangrene, soft tissue infections, radiation-induced necrosis, crush syndrome, osteomyelitis, postoperative neurosurgical infection, and postoperative discitis $[11,12]$. We therefore speculated that HBO therapy would be effective when pyogenic spondylitis is the primary focus of infection. We have now become more experienced in treating pyogenic spondylitis accompanied by iliopsoas abscess using percutaneous drainage combined with $\mathrm{HBO}$ therapy. To the best of our knowledge, there have been no previous reports of the use of percutaneous drainage combined with $\mathrm{HBO}$ therapy for the treatment of this condition, and the purpose of this article is to evaluate the outcomes in patients who have undergone this therapy.

\section{Materials and Methods}

\section{Patients}

Twenty-three patients with pyogenic spondylitis accompanied by iliopsoas abscess were treated using percutaneous drainage combined with $\mathrm{HBO}$ therapy in addition to common conservative therapy, including bed rest and pharmacotherapy with antibiotics and/or immunoglobulin preparations, in our hospital between April 2004 and December 2011. There were 13 men and 10 women (mean age, 69.0 years; range, $45-85$ years). The mean duration of follow-up was 27.7 months (range, 12-48 months). The indications for use of this method to treat pyogenic spondylitis with iliopsoas abscess were as follows: 1) involved vertebra located in the lumbar spine; 2 ) absence of major neurological deficits; 3 ) absence of radiographically remarkable bone destruction of the affected vertebral body (we included cases with signal intensity change in the vertebral body on magnetic resonance imaging [MRI] and/or endplate collapse and excluded cases with vertebral body collapse); and 4) abscess measuring $\geq 30 \mathrm{~mm}$ on the axial view of the MRI. Of the 23 patients, 22 experienced low back pain. Four patients exhibited mild or moderate radicular pain, and 1 patient had left hip pain. No patient had bowel or bladder dysfunction. The affected intervertebral discs were L1-2 in 4 patients, L2-3 in 6 patients, L3-4 in 5 patients, L4-5 in 7 patients, and L5-S1 in 1 patient. The total number of iliopsoas abscesses was 29 , affecting the right side in 6 patients, the left side in 11 patients, and both sides in 6 patients. The abscesses were measured at their largest dimensions on the MRI axial view. The mean size of the abscesses was $48.1 \mathrm{~mm}$ (range, 30.0-79.1 mm). Mean C-reactive protein (CRP) level at admission was $12.83 \mathrm{mg} / \mathrm{dL}$ (range, $1.78-23.76 \mathrm{mg} / \mathrm{dL}$ ). Comorbidity included diabetes mellitus in 7 patients, renal failure in 3 patients, carcinoma in 2 patients, urinary tract infection in 2 patients, pneumonia in 1 patient, and bronchial asthma in 1 patient (Table 1).

\section{Description of the drainage procedures}

The patients were positioned on the computed tomography (CT) table with markers (Webb Manufacturing Co., Philadelphia, PA, USA) attached to the skin. A preprocedural CT scan was obtained to confirm the location and size of the abscess and its relation to nearby structures so that a safe and proper route could be selected and to determine of the angle and depth of puncture. After the skin was disinfected, a disposable 20 -cm-long 22-gauge puncture needle (Create Medic Co., Yokohama, Japan) was inserted under local anesthesia and advanced to the center of the iliopsoas abscess, with monitoring of the needle tip by CT as needed. A guidewire was then passed 
Table 1. Summary of patient characteristics

\begin{tabular}{|c|c|}
\hline \multicolumn{2}{|l|}{ Characteristics } \\
\hline Age (yr) & $69.0(45-85)$ \\
\hline \multicolumn{2}{|l|}{ Sex } \\
\hline Male & 13 \\
\hline Female & 10 \\
\hline \multicolumn{2}{|l|}{ Symptoms } \\
\hline Low back pain & 22 \\
\hline Radicular pain & 4 \\
\hline Hip pain & 1 \\
\hline \multicolumn{2}{|l|}{ Affected intervertebral discs } \\
\hline L1-2 & 4 \\
\hline L2-3 & 6 \\
\hline L3-4 & 5 \\
\hline L4-5 & 7 \\
\hline L5-S1 & 1 \\
\hline \multicolumn{2}{|l|}{ Abscess location } \\
\hline Right side & 6 \\
\hline Left side & 11 \\
\hline Both sides & 6 \\
\hline Size of abscesses (mm) & $48.1(30.0-79.1)$ \\
\hline CRP level at admission (mg/dL) & $12.83(1.78-23.76)$ \\
\hline \multicolumn{2}{|l|}{ Underlying disease } \\
\hline Diabetes mellitus & 7 \\
\hline Renal failure & 3 \\
\hline Carcinoma & 2 \\
\hline Urinary tract infection & 2 \\
\hline Pneumonia & 1 \\
\hline Bronchial asthma & 1 \\
\hline
\end{tabular}

Values are presented as mean (range) or number.

CRP, C-reactive protein. through the outer sheath of the puncture needle into the abscess. The insertion point was adequately enlarged with a dilator and the drainage catheter inserted over the guidewire. After complete evacuation of the abscess by aspiration, the drainage catheter was affixed to the skin (Fig. 1).

\section{HBO treatment}

In accordance with our protocol, patients were scheduled to receive $\mathrm{HBO}$ therapy $\left(100 \% \mathrm{O}_{2}\right.$ at 2.5 atmosphere absolute for 90 minutes) once daily for 20 consecutive days. If there was evidence of significant clinical and laboratory improvement, the $\mathrm{HBO}$ treatments were discontinued. In contrast, if the patient had not recovered sufficiently by the 20th $\mathrm{HBO}$ session, additional $\mathrm{HBO}$ treatments were recommended.

\section{Clinical evaluation}

After the procedure, all patients underwent routine clinical, laboratory, and imaging examinations. Radiographs were checked for signs of bone destruction such as vertebral collapse and spinal deformities such as kyphosis and scoliosis. Contrast-enhanced MRI or CT was used to investigate the abscess size and search for phlegmon. The following parameters were retrospectively evaluated: 1) alleviation of symptoms; 2) changes in inflammatory markers on laboratory tests; 3 ) changes in the findings of radiographic imaging, including bone destruction, spinal deformity, and instability; 4) change in the abscess size; 5) recurrence rate; and 6) clinical outcome at the final follow-up. The clinical outcome was graded as excellent
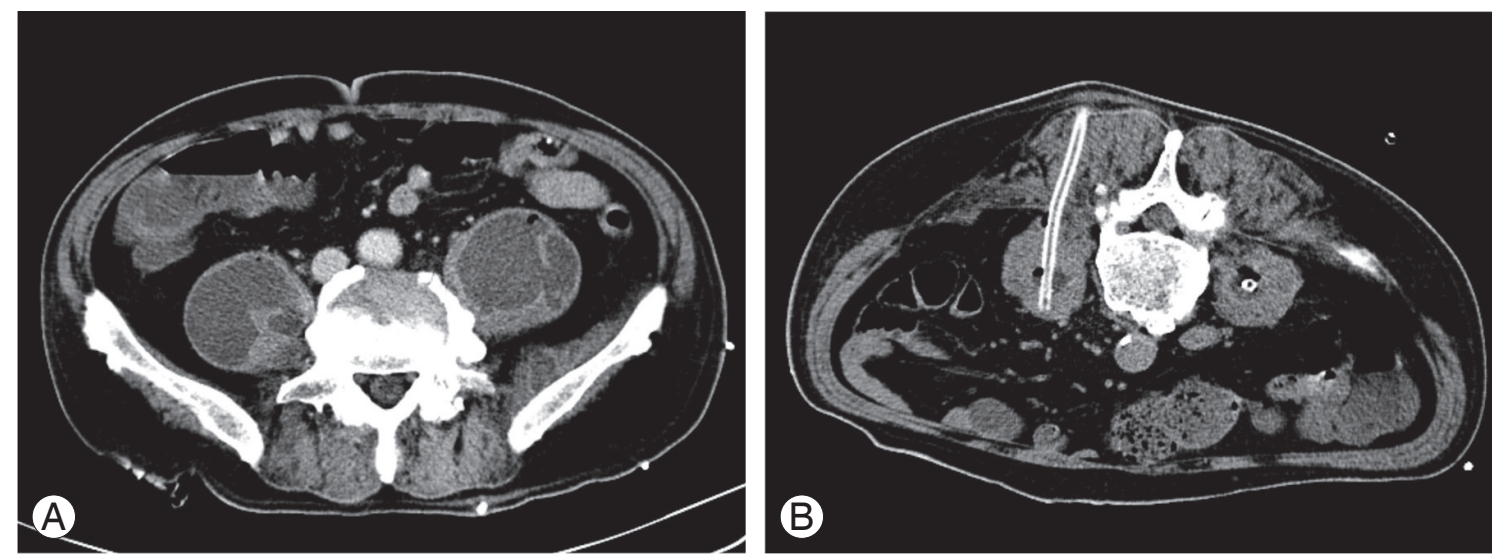

Fig. 1. (A) Axial computed tomography scan, obtained before drainage, showing bilateral iliopsoas abscesses. (B) Axial computed tomography scan obtained after the insertion of drainage tubes followed by evacuation of the abscess by aspiration. 
when there was no pain and no limitation in activities of daily living (ADLs); good if there was no limitation in ADLs but mild occasional pain; fair in the cases with slight limitations in ADLs and continuing pain that was not as great as that before the procedure; and poor in cases with limitations in ADLs and persistent, marked pain or no response to the procedure.

\section{Results}

All 23 patients were treated with percutaneous drainage combined with $\mathrm{HBO}$ therapy in addition to common conservative therapy including bed rest and pharmacotherapy with antibiotics and/or immunoglobulin preparations. The pathogens were identified from collected samples in 16 of the 23 patients. Mean duration of drainage was 21.1 days (range, 5-62 days). CT-guided percutaneous insertion of the drainage tube into the iliopsoas abscess was successful in all patients, and there were no procedural complications. The average total cycle of $\mathrm{HBO}$ treatments for each patient was 19.3 days (range, 5-47 days). For 15 patients (65.5\%), HBO treatments ended within 20 cycles; for 8 patients (34.7\%), HBO treatments ended at over 20 cycles. There were no major side effects, such as cerebral oxygen toxicity or adverse effects of pressurization associated with $\mathrm{HBO}$ treatment. Low back pain in 22 patients, radicular pain in 4 patients, and hip pain in 1 patient resolved immediately after treatment. The mean time required from the start of treatment for CRP to decrease to normal levels or to reach the level before the onset of spondylitis was 28.3 days (range, 8-56 days). Progressive destructive changes were absent in the final follow-up radiographic studies in all patients. No patient displayed progression of spinal deformity or instability. Follow-up MRI or CT with enhancement confirmed the disappearance or near-total resolution of the iliopsoas abscess cavity and healing of the pyogenic spondylitis in all 23 patients. No recurrence was observed in any patient at follow-up. At the final follow-up, the clinical outcomes were rated as excellent in 11 patients, good in 9 patients, and fair in 3 patients. There were no patients with poor outcomes according to the classification described (Table 2).

\section{Discussion}

Conservative therapy with antibiotics is generally the initial
Table 2. Summary of patient outcomes

\section{Outcomes}

\begin{tabular}{|cc}
\hline Identified pathogen & 6 \\
\hline Staphylococcus aureus & 4 \\
\hline MRSA & 2 \\
\hline Streptococcus pneumoniae & 1 \\
\hline Staphylococcus epidermidis & 1 \\
\hline Klebsiella pneumoniae & 1 \\
\hline Bacteroides fragilis & 1 \\
\hline Escherichia coli & $21.1(5-62)$ \\
\hline Duration of drainage (day) & $19.3(5-47)$ \\
\hline Number of HBO treatments & 23 \\
\hline Resolution of symptoms & $28.3(8-56)$ \\
\hline Normalization of CRP (day) & 0 \\
\hline Radiographic findings & 0 \\
\hline Progressive destruction & 0 \\
\hline Progression of spinal deformity & 0 \\
\hline Spinal instability & 3 \\
\hline Recurrence & 0 \\
\hline Clinical outcomes & 11 \\
\hline Excellent & 9 \\
\hline Good & \\
\hline Fair & \\
\hline Poor & \\
\hline
\end{tabular}

Values are presented as number or mean (range).

MRSA, methicillin-resistant Staphylococcus aureus; HBO, hyperbaric oxygen; CRP, C-reactive protein.

treatment of choice for pyogenic spondylitis. However, because of the increased number of patients who are immunosuppressed due to the effects of certain medications or underlying diseases, it is becoming more difficult to treat patients with pyogenic spondylitis using only common conservative therapy. Open surgery is required in cases of pyogenic spondylitis in which conservative treatment is ineffective or in which advanced bone destruction or severe neurological deficits are present. There have been a number of reports regarding surgical options for pyogenic spondylitis. Some authors have suggested that favorable results are best achieved using anterior curettage and autologous bone grafting $[13,14]$, and Wisneski [15] has also included increased paraspinal abscess formation among the indications for open surgery. In addition, Malawski and Lukawski [16] have reported that although small paraspinal abscesses usually respond 
to conservative treatment, larger paraspinal abscesses can be treated surgically with successful results. However, in 147 surgically treated patients, 4 cases of mortality due to cardiac or renal disease and secondary pneumonia were encountered. Because patients with pyogenic spondylitis who require surgery are often immunocompromised and in poor general condition, less invasive operative procedures are desirable.

Percutaneous drainage of iliopsoas abscess under CT guidance may be a recognized alternative to surgery, but in patients with secondary iliopsoas abscess without radical treatment of the primary focus of infection (i.e., spondylitis), drainage of the iliopsoas abscess alone is sometimes not enough. For patients in whom spondylitis is the primary focus of infection, prolonged infection of a vertebral body or intervertebral disc usually leads to spinal deformity or instability, resulting in the need for surgical intervention. Without intervention for the primary focus, a prolonged period of therapy may be required, and there can be recurrence even if the infection is initially cured. We have previously reported that the mean time required for normalization of CRP is 30.2 days for treatment consisting of continuous irrigation via a saline infusion tube inserted in the infected disc and a drainage tube in the secondary iliopsoas abscess [9]. The mean time required for normalization of CRP was 28.3 days in the present study, which is comparable to the data of our previous study with a similar disease stage and patient background; thus, there was no prolonged period of therapy in this study. Several authors have previously reported recurrence of iliopsoas abscesses after performing percutaneous drainage alone without curative treatment of the primary focus of infection. Dinc et al. [2] reported that $6(29 \%)$ of 21 patients had recurrences within 1 to 3 months after catheter removal in their study of percutaneous drainage of iliopsoas and spondylodiscitic abscesses measuring $>3 \mathrm{~cm}$. Yacoub et al. [3] reported a recurrence rate of $15 \%$ (4 of 26 patients) after percutaneous drainage of psoas abscess. Tabrizian et al. [4] reported that the failure rate of percutaneous drainage alone was as high as $60 \%$ ( 29 of 48 patients) and that $44 \%$ (21 of 48 ) of patients ultimately required operative management. Cronin et al. [5] reported that $10(10 \%)$ of 99 patients had recurrent abscesses after catheter removal. Therefore, percutaneous drainage of iliopsoas abscesses alone might not be an established treatment for spondylitis accompanied by iliopsoas abscesses. Some investigators consider it essen- tial to combine abscess drainage with curative treatment of the primary focus of infection [6], and we believe that a more effective means of preventing a prolonged period of therapy and recurrence is a crucial factor in successful and less invasive treatment.

Several minimally invasive techniques for the treatment of spondylitis have recently been described. Jeanneret and Magerl [17] used percutaneous external fixation after percutaneous suction or irrigation. Haaker et al. [18] performed percutaneous lumbar discectomy for 16 patients with lumbar disc infection and reported satisfactory results. Nagata et al. [19] performed percutaneous suction aspiration with drainage in 23 cases of early-stage pyogenic spondylitis and demonstrated the effectiveness of this procedure. Hadjipavlou et al. [20] reported percutaneous transpedicular discectomy and drainage in pyogenic spondylodiscitis. However, all of these procedures were effective in the early stages of spondylitis and may not be useful in patients with advanced spondylitis, such as those with a large paraspinal abscess measuring $\geq 30$ $\mathrm{mm}$ on axial MRI views.

Several studies have reported on the treatment of spondylitis that is accompanied by large iliopsoas abscesses using less invasive treatments directed at the spondylitis as the primary focus of infection. Hanaoka et al. [7] performed percutaneous drainage and continuous irrigation of the infected disc space in 5 patients with severe pyogenic spondylitis with abscess formation or marked bone destruction and demonstrated successful results. Ito et al. [8] reported good clinical results for posterolateral endoscopic debridement with irrigation in a study of 15 patients with pyogenic spondylodiscitis, including 6 patients with psoas abscesses. We have previously reported that in 12 patients with pyogenic spondylitis accompanied by iliopsoas abscess, continuous irrigation using a saline infusion tube inserted in the infected disc and a drainage tube in the secondary iliopsoas abscess communicating with the primary lesion was effective [9]. Matsumoto et al. [10] reported that CT-guided percutaneous drainage within the intervertebral space was effective for pyogenic spondylodiscitis with psoas abscess in a study of 8 patients. Although all the above-mentioned procedures were effective and produced good clinical results, they may still be technically demanding, time-consuming, or complicated, which can make it difficult for them to become widely established in routine clinical practice.

$\mathrm{HBO}$ is used in the management of a variety of in- 
fected, hypoperfused, and hypoxic wounds. HBO plays an important role in stimulation of the bactericidal action of white blood cells [11]. The bactericidal capacity of leukocytes is substantially impaired at the low oxygen tension observed in wounds [21,22]. HBO therapy increases the oxygen tension in infected tissues, resulting in direct bactericidal effects on some organisms. Improved tissue oxygen tension in ischemic tissues during HBO therapy inhibits the growth of aerobic and facultative anaerobic bacteria. These effects are mediated in large part by oxygen-based free radicals that oxidize proteins and membrane lipids, damage DNA, and inhibit metabolic functions essential for microbial growth [23]. HBO therapy also stimulates neovascularization, thus decreasing tissue ischemia. These angiogenic effects are dose-dependent and can result in 8- to 9-fold increases in the vascular density of tissues [24]. Improved vascularity not only improves tissue oxygen tension and host defenses but also facilitates entry of leukocytes, antibodies, and antibiotics into the infected lesion. In addition, HBO therapy stimulates fibroplasia and bone remodeling [25]. We have found that the addition of $\mathrm{HBO}$ treatment to percutaneous drainage of iliopsoas abscess can lead to a cure when spondylitis is the primary focus of infection. Therefore, we believe that percutaneous drainage combined with $\mathrm{HBO}$ therapy is an effective and reasonable treatment for spondylitis accompanied by a large iliopsoas abscess.

\section{Conclusions}

The findings of the present study suggest that patients with pyogenic spondylitis accompanied by a iliopsoas abscess can be cured without a prolonged period of therapy or recurrence by using this treatment. We believe that CT-guided percutaneous drainage of iliopsoas abscesses, which is a less invasive and not technically demanding method of therapy, can become an established treatment if it is combined with $\mathrm{HBO}$ therapy.

\section{Conflict of Interest}

No potential conflict of interest relevant to this article was reported.

\section{References}

1. Staatz G, Adam GB, Keulers P, Vorwerk D, Gunther
RW. Spondylodiskitic abscesses: CT-guided percutaneous catheter drainage. Radiology 1998;208:363-7.

2. Dinc H, Ahmetoglu A, Baykal S, Sari A, Sayil O, Gumele HR. Image-guided percutaneous drainage of tuberculous iliopsoas and spondylodiskitic abscesses: midterm results. Radiology 2002;225:353-8.

3. Yacoub WN, Sohn HJ, Chan S, et al. Psoas abscess rarely requires surgical intervention. Am J Surg 2008; 196:223-7.

4. Tabrizian P, Nguyen SQ, Greenstein A, Rajhbeharrysingh U, Divino CM. Management and treatment of iliopsoas abscess. Arch Surg 2009;144:946-9.

5. Cronin CG, Gervais DA, Hahn PF, Arellano R, Guimaraes AR, Mueller PR. Treatment of deep intramuscular and musculoskeletal abscess: experience with 99 CT-guided percutaneous catheter drainage procedures. AJR Am J Roentgenol 2011;196:1182-8.

6. Muckley T, Schutz T, Kirschner M, Potulski M, Hofmann G, Buhren V. Psoas abscess: the spine as a primary source of infection. Spine (Phila Pa 1976) 2003;28:E106-13.

7. Hanaoka N, Kawasaki Y, Sakai T, et al. Percutaneous drainage and continuous irrigation in patients with severe pyogenic spondylitis, abscess formation, and marked bone destruction. J Neurosurg Spine 2006;4: 374-9.

8. Ito M, Abumi K, Kotani Y, Kadoya K, Minami A. Clinical outcome of posterolateral endoscopic surgery for pyogenic spondylodiscitis: results of 15 patients with serious comorbid conditions. Spine (Phila Pa 1976) 2007;32:200-6.

9. Tofuku K, Koga H, Yone K, Komiya S. Continuous irrigation in pyogenic spondylitis accompanied by iliopsoas abscess. Spine (Phila Pa 1976) 2007;32:E382-7.

10. Matsumoto T, Yamagami T, Morishita H, et al. CTguided percutaneous drainage within intervertebral space for pyogenic spondylodiscitis with psoas abscess. Acta Radiol 2012;53:76-80.

11. Larsson A, Engstrom M, Uusijarvi J, Kihlstrom L, Lind F, Mathiesen T. Hyperbaric oxygen treatment of postoperative neurosurgical infections. Neurosurgery 2002;50:287-95.

12. Kutlay M, Colak A, Simsek H, et al. Antibiotic and hyperbaric oxygen therapy in the management of post-operative discitis. Undersea Hyperb Med 2008; 35:427-40.

13. Fang D, Cheung KM, Dos Remedios ID, Lee YK, Le- 
ong JC. Pyogenic vertebral osteomyelitis: treatment by anterior spinal debridement and fusion. J Spinal Disord 1994;7:173-80.

14. Przybylski GJ, Sharan AD. Single-stage autogenous bone grafting and internal fixation in the surgical management of pyogenic discitis and vertebral osteomyelitis. J Neurosurg 2001;94(1 Suppl):1-7.

15. Wisneski RJ. Infectious disease of the spine. Diagnostic and treatment considerations. Orthop Clin North Am 1991;22:491-501.

16. Malawski SK, Lukawski S. Pyogenic infection of the spine. Clin Orthop Relat Res 1991;(272):58-66.

17. Jeanneret B, Magerl F. Treatment of osteomyelitis of the spine using percutaneous suction/irrigation and percutaneous external spinal fixation. J Spinal Disord 1994;7:185-205.

18. Haaker RG, Senkal M, Kielich T, Kramer J. Percutaneous lumbar discectomy in the treatment of lumbar discitis. Eur Spine J 1997;6:98-101.

19. Nagata K, Ohashi T, Ariyoshi M, Sonoda K, Imoto H, Inoue A. Percutaneous suction aspiration and drainage for pyogenic spondylitis. Spine (Phila Pa 1976)
1998;23:1600-6.

20. Hadjipavlou AG, Katonis PK, Gaitanis IN, Muffoletto AJ, Tzermiadianos MN, Crow W. Percutaneous transpedicular discectomy and drainage in pyogenic spondylodiscitis. Eur Spine J 2004;13:707-13.

21. Allen DB, Maguire JJ, Mahdavian M, et al. Wound hypoxia and acidosis limit neutrophil bacterial killing mechanisms. Arch Surg 1997;132:991-6.

22. Mader JT, Brown GL, Guckian JC, Wells CH, Reinarz JA. A mechanism for the amelioration by hyperbaric oxygen of experimental staphylococcal osteomyelitis in rabbits. J Infect Dis 1980;142:915-22.

23. Jamieson D, Chance B, Cadenas E, Boveris A. The relation of free radical production to hyperoxia. Annu Rev Physiol 1986;48:703-19.

24. Marx RE, Ehler WJ, Tayapongsak P, Pierce LW. Relationship of oxygen dose to angiogenesis induction in irradiated tissue. Am J Surg 1990;160:519-24.

25. Brismar K, Lind F, Kratz G. Dose-dependent hyperbaric oxygen stimulation of human fibroblast proliferation. Wound Repair Regen 1997;5:147-50. 